
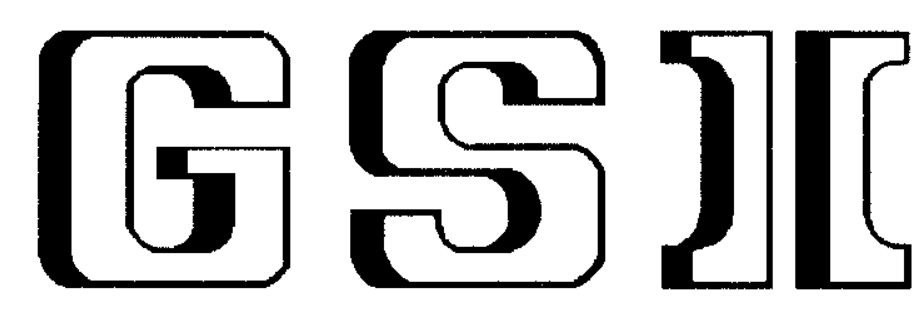

GSI-Preprint-99-32

September 1999

\title{
IMPACT-PARAMETER DEPENDENCE OF GIANT RESONANCE EXCITATIONS IN RELATIVISTIC HEAVY-ION COLLISIONS
}

A. Grünschloß, K. Boretzky, T. Aumann, C.A. Bertulani, J. Cub, W. Dostal, B. Eberlein, Th.W. Elze, H. Emling, J. Holeczek, R. Holzmann, M. Kaspar, J.V. Kratz, R. Kulessa, Y. Leifels, A. Leistenschneider, E. Lubkiewicz, S. Mordechai, I. Peter, P. Reiter, M.

Rejmund, H. Simon, K. Stelzer, A. Surowiec, K. Sümmerer, J. Stroth, E. Wajda, W. Walus, S. Wan, H.J. Wollersheim

Gesellschaft für Schwerionenforschung $\mathrm{mbH}$ Planckstraße 1 • D-64291 Darmstadt • Germany Postfach 110552 • D-64220 Darmstadt • Germany

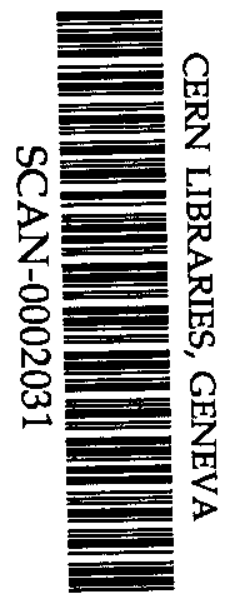




\title{
Impact-Parameter Dependence of Giant Resonance Excitations in Relativistic Heavy-Ion Collisions
}

A. Grünschloß ${ }^{1}$, K. Boretzky ${ }^{2}$, T. Aumann ${ }^{2,3}$, C.A. Bertulani ${ }^{4}$, J. Cub ${ }^{3,5}$, W. Dostal ${ }^{2}$, B. Eberlein ${ }^{2}$, Th.W. Elze $^{1}$, H. Emling ${ }^{3}$, J. Holeczek ${ }^{3}$, R. Holzmann ${ }^{3}$, M. Kaspar ${ }^{3}$, J.V. Kratz ${ }^{2}$, R. Kulessa ${ }^{6}$, Y. Leifels ${ }^{3}$, A. Leistenschneider ${ }^{1}$, E. Lubkiewicz ${ }^{6}$, S. Mordechai ${ }^{7}$, I. Peter ${ }^{3}$, P. Reiter ${ }^{8}$, M. Rejmund ${ }^{3}$, H. Simon ${ }^{5}$, K. Stelzer ${ }^{1}$, A. Surowiec ${ }^{3}$, K. Sümmerer ${ }^{3}$, J. Stroth ${ }^{1}$, E. Wajda ${ }^{6}$, W. Walus ${ }^{6}$, S. Wan ${ }^{3}$, and H.J. Wollersheim ${ }^{3}$

${ }^{1}$ Institut für Kernphysik, Johann-Wolfgang-Goethe-Universität, D-60486 Frankfurt, Germany

${ }^{2}$ Institut für Kernchemie, Johannes Gutenberg-Universität, D-55099 Mainz, Germany

${ }^{3}$ Gesellschaft für Schwerionenforschung (GSI), Planckstr. 1, D-64291 Darmstadt, Germany

${ }^{4}$ Instituto de Física, Universidade Federal, 21945-970 Rio do Janeiro, Brazil

${ }^{5}$ Institut für Kernphysik, Technische Universität, D-64289 Darmstadt, Germany

${ }^{6}$ Instytut Fizyki, Uniwersytet Jagielloniski, PL-30-059 Kraków, Poland

${ }^{7}$ Ben-Gurion University of the Negev, Beer-Sheva, Israel

${ }^{8}$ Max Planck Institut für Kernphysik, D-69029 Heidelberg, Germany

(August 30, 1999)

\begin{abstract}
Angular distributions of Xe fragments produced in peripheral collisions of a ${ }^{136} \mathrm{Xe}$ beam (700 $\mathrm{MeV} /$ nucleon) with ${ }^{208} \mathrm{~Pb}$ and ${ }^{\text {nat }} \mathrm{Sn}$ targets were measured. Equivalent sharp-cutoff minimum impact parameters were derived on the basis of a semi-classical description for the electromagnetic excitation of one- and two-phonon giant resonances. The results are compared with current standard parametrizations of minimum impact parameters and with the soft-spheres model using realistic mass density distributions for projectile and targets.
\end{abstract}

PACS numbers: $25.70 . \mathrm{De}, 24.30 . \mathrm{Cz}, 27.60 .+\mathrm{j}, 23.20 . \mathrm{En}$

Giant resonances, in particular the isovector giant dipole resonance, are Coulomb excited with large cross sections in peripheral heavy-ion collisions at bombarding energies of several hundred $\mathrm{MeV} /$ nucleon and above. One of the most interesting features is the possibility to study double-phonon giant dipole resonances (DGDR), to which a number of experimental studies were devoted. The basic characteristics of the DGDR are now fairly well explored. To a large extent, the observations are in reasonable agreement with the theoretical concept describing giant dipole resonance (GDR) phonons as almost harmonic quantized vibrations [1]. Yet, persisting discrepancies are found as far as the DGDR cross sections are concerned, which are enhanced up to about a factor 2 compared with the harmonic phonon picture. The enhancement is particularly pronounced in the case of ${ }^{136} \mathrm{Xe}[2]$, the nucleus under investigation in this experiment. For a detailed review of the status of multi-phonon giant resonances we refer to [1].

Usually, measured cross sections are compared with semi-classical calculations which adopt classical trajectories of the scattered ion, while the process of electromagnetic excitation is treated quantum-mechanically $[3,4]$. The trajectory is determined by its impact parameter $b$. For Coulomb trajectories in high-energy collisions, the extremely small scattering angles $\theta$ of the ion in the laboratory frame are related to $b$ as

$$
\theta=\frac{2 Z_{p} Z_{t} e^{2}}{b v^{2} \gamma m_{p}}
$$

$Z_{p}$ and $Z_{t}$ denote the nuclear charge of the collision partners, $m_{p}$ the projectile mass, $v$ the projectile velocity, and $\gamma$ the Lorentz factor. Up to now, only angle-integrated cross sections are experimentally available. Here, we report on results of a first measurement of fragment angular distributions, performed in order to check the validity of the semi-classical approach.

Measurements of angle-differential cross sections are difficult to achieve. The relevant scattering angles amount to a few milliradians only, and multiple scattering to small angles (angular straggling) in relatively thick targets, as usually applied, washes out the angular distributions considerably. One of the problems in analyzing double-phonon GDR data is how to treat effects due to nuclear interactions. For impact parameters around grazing incidence, electromagnetic cross sections are reduced due to nuclear reactions absorbing part of the flux. Most frequently, the electromagnetic cross sections are computed adopting a minimum impact parameter $b_{\min }$ below which electromagnetic excitation is cut off. This 'sharp-cutoff' approximation was contrasted with a smooth cutoff, the 'soft-spheres model' [5], which will be discussed below. Several (semi-) empirical parametrizations for $b_{\min }$ exist. In analyses of experimental data and in theoretical approaches, most commonly, the parametrization given by Benesh, Cook, and Vary (BCV) [6] is adopted. Occasionally, another parametrization given by Kox et al. [7] is used which yields somewhat larger values of $b_{\min }$.

An appropriate choice of $b_{\min }$ is crucial in calculating reliable giant resonance cross sections. The largest fraction of the double-phonon GDR cross section, in particular, is obtained from trajectories with impact parameters near grazing incidence. Thus, an inaccurate choice of 
$b_{\min }$ could strongly influence observed two-phonon cross section enhancement factors as extracted from experiments. For instance, the two above parametrizations [6] and [7] of $b_{\min }$ yield DGDR cross sections differing by about $40 \%$ for ${ }^{136} \mathrm{Xe}(700 \mathrm{MeV} /$ nucleon $)$ excited on a $\mathrm{Pb}$ target.

For this reason we performed a measurement of the scattering-angle (or impact-parameter) dependence of giant resonance cross sections in near-relativistic heavy-ion collisions. Since the largest DGDR cross section enhancement factor was observed for ${ }^{136} \mathrm{Xe}[2]$, we choose this nucleus. The experiment, in general, served as well to re-examine the results of this earlier measurement, to be reported in a forthcoming publication. Here, we shall focus on an analysis of the impact-parameter dependence of cross sections.

A ${ }_{54}^{136} \mathrm{Xe}$ beam in the $46+$ atomic charge state was accelerated by the SIS synchrotron at GSI, Darmstadt, to an energy of $700 \mathrm{MeV} /$ nucleon. After extraction, the beam passed a diaphragm with a $1 \mathrm{~mm}$ circular aperture. Ions hitting the diaphragm material became essentially fully stripped and were removed from the beam by deflecting magnets within the transport line to the experimental area. In this way, the beam emittance was reduced substantially. The ${ }^{136} \mathrm{Xe}$ projectiles were directed onto a ${ }^{208} \mathrm{~Pb}\left(54 \mathrm{mg} / \mathrm{cm}^{2}\right)$, ${ }^{\text {nat }} \mathrm{Sn}\left(239 \mathrm{mg} / \mathrm{cm}^{2}\right)$, or ${ }^{\text {nat }} \mathrm{C}$ $\left(274 \mathrm{mg} / \mathrm{cm}^{2}\right)$ target. The trajectories of the incoming and scattered projectiles and projectile fragments were traced as described in detail below. The nuclear charge of the projectile fragments was determined by an energyloss measurement in two silicon diodes placed behind the target. Nuclear masses of the fragments were obtained from their magnetic rigidities and their velocities. For that purpose, the fragments were deflected in a large-gap dipole magnet, and the deflection angles measured with two arrays of scintillating fibers (1 mm pitch) coupled to position sensitive phototubes (for details see [8]). An array of thin organic plastic scintillators allowed for a time-of-flight measurement over a distance of $13.7 \mathrm{~m}$. In order to minimize angular straggling effects, the heavy ions were kept in vacuum throughout their travel until reaching the first fiber array.

The fragments were detected in coincidence with neutrons emitted in forward direction by means of the LAND detector [9] and with $\gamma$ rays by means of the NaI CrystalBall spectrometer [10] covering essentially the full solid angle around the target. In heavy nuclei, giant resonances decay almost exclusively by neutron evaporation and subsequent $\gamma$ decay of the residual fragments. Thus, giant resonance excitations were selected by requiring that the nuclear charge of the Xe projectiles remains unchanged and that the process is accompanied by neutron cmission. The number of detected neutrons (up to four neutrons) was required to match the mass difference between the Xe projectile and its fragment. This condition suppresses to a large extent competing nucleon-knockout reactions which are accompanied by nucleons scattered to angles beyond the acceptance of the neutron detector. In contrast, neutrons from giant resonance decay are kinematically strongly focussed in forward direction and are fully covered by the LAND angular acceptance. In case of the extremely thin $\mathrm{Pb}$ target, an additional coincidence with $\gamma$ rays in the $\mathrm{NaI}$ spectrometer was required in order to further suppress background originating from interactions of projectiles outside the target. Incident projectiles were monitored event-by-event in a downscaled mode without requiring any coincidence. This enables one to determine absolute cross sections after applying corrections for detector efficiencies and acceptances. We estimate a systematic error of $15 \%$ for the absolute normalization of the cross sections. The residual background due to interactions outside the target which still obeyed the above trigger conditions, was determined from a measurement without target and could be subtracted. It is localized at scattering angles below $1.5 \mathrm{mrad}$ and contributs on a level of about $10 \%$. In order to obtain an estimate of contributions from nuclear reactions, a measurement using a carbon target was performed. Also, reactions were analyzed which yield fragments other than Xe, unlikely to be produced via giant resonance excitations and subsequent decay. The experimental technique, although improved considerably, and the concept of the method, in essence, are the same as in an earlier study of the DGDR in ${ }^{208} \mathrm{~Pb}$ [11] to which we refer for details.

The essential part of the measurement reported here consists of an accurate determination of the laboratory scattering angles of the projectile fragments. The beam, narrowed down in emittance as mentioned above, first passed a thin $\left(25 \mathrm{mg} / \mathrm{cm}^{2}\right)$ organic plastic counter located $10.1 \mathrm{~m}$ upstream from the target. The detector of $5 \mathrm{~cm}$ by $5 \mathrm{~cm}$ size was coupled to 4 phototubes at its four edges. From the relative light output observed in the phototubes, the position of incidence could be determined in both directions $(x, y)$ perpendicular to the beam with a resolution $\sigma_{x, y}=0.5 \mathrm{~mm}$. This detector also served to produce a start signal of the time-of-flight measurement, see above. Instead of placing a second position sensitive detector in front of the target (in order to determine the projectile trajectory) we used an active 4-jaw beam collimator placed $1.5 \mathrm{~m}$ in front of the target. Projectiles hitting the collimator, made of organic plastic scintillators coupled to phototubes, were rejected. The aperture was chosen to be $1 \mathrm{~mm}$ by $1 \mathrm{~mm}$ ( $3 \mathrm{~mm}$ by $3 \mathrm{~mm}$ ) in case of the the $\mathrm{Pb}(\mathrm{Sn})$ target. In this way we avoided straggling effects outside the target. The $(x, y)$ positions of the projectile fragments were determined by means of two single-sided Si-strip detectors of $100 \mu \mathrm{m}$ pitch and $150 \mu \mathrm{m}$ thickness placed $1.0 \mathrm{~m}$ downstream from the target. Using projectiles which did not undergo a reaction in the target, the relative positions of the collimator and the detectors to each other could be determined with an accuracy corresponding to $0.1 \mathrm{mrad}$ with regard to scat- 
tering angle. The resolution with regard to scattering angle was inspected by event simulation taking into account the positional accuracies as quoted above and also the multiple scattering in the target itself. The angular straggling and the size of the collimator aperture contribute most to the overall angular resolution. Other effects, e.g. a modification of the fragment scattering angle due to neutron evaporation, can safely be neglected. The resulting distributions describing the resolution with regard to scattering angle can be approximated fairly well by gaussian distributions with a width of $\sigma_{\theta}=0.62(1.20)$ mrad for the $\mathrm{Pb}(\mathrm{Sn})$ target.

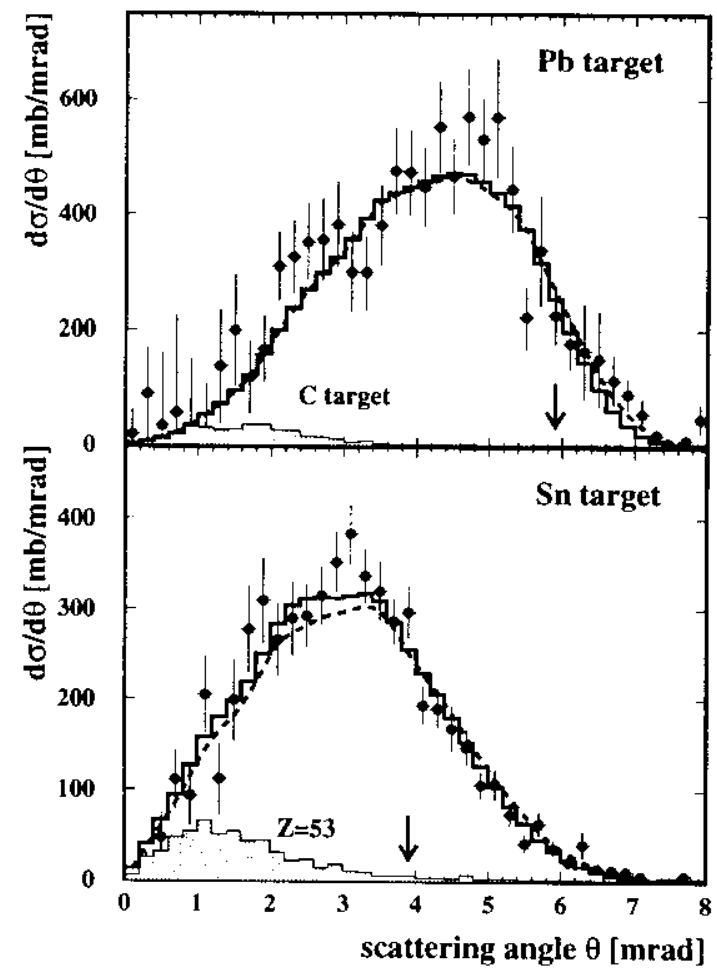

FIG. 1. Top: Differential cross section $d \sigma / d \theta$ for Xe fragments and the ${ }^{208} \mathrm{~Pb}$ (upper panel) and ${ }^{n a t} \mathrm{Sn}$ (lower panel) target. Results of semi-classical calculations using the best choice (see Tab. I) of sharp-cutoff minimum impact parameters $b_{\min }$ are shown by the histograms, the results using the soft-spheres model by the solid curves. The arrows indicate the laboratory scattering angles corresponding to the $b_{m i n}$ values (see Eq. 1). The shaded areas indicate the cross section obtained with the $\mathrm{C}$ target (upper panel) and that for fragments of nuclear charge $Z=53$ (lower panel).

Fig. 1 shows the Xe fragment angular distribution in the laboratory frame $d \sigma / d \theta$ as measured with the $\mathrm{Pb}$ and Sn targets. In order to get an estimate of contributions from nuclear interactions, a similar analysis was performed for fragments undergoing charge changing reactions (see above). Fig. 1 incorporates the angular distribution for fragments of nuclear charge $Z=53$ in case of the $S_{n}$ target, revealing a small contribution which is even smaller in case of the $\mathrm{Pb}$ target. One also recognizes that the nuclear cross section is located at angles below that of grazing incidence in Coulomb scattering, which is of most interest in the current data analysis. Likewise, the cross sections obtained from the measurement with the $\mathrm{C}$ target, analyzed under equivalent constraints as for the $\mathrm{Pb}$ and $\mathrm{Sn}$ target, were also found to be small, see Fig. 1.

The evaluation of the minimum impact parameter proceeds as follows: We compute the impact-parameterdependent cross sections for the electric isovector giant dipole resonance and isoscalar and isovector quadrupole resonances in semi-classical approximation. The minimum impact parameter $b_{\min }$ is varied as it is the variable to be determined. The cross section for the twophonon dipole resonance is obtained by applying the folding model described in [12], on the basis of the harmonic phonon picture. Contributions from resonances of higher multipolarity or magnetic resonances can be neglected. In these calculations we use strength distributions extracted from photoabsorption measurements [13] in case of the dipole resonance and from the systematics of data in case of the quadrupole resonances [14] (for details see [2]). The cross section of the single-phonon dipole resonance dominates, the other resonances contribute typically $15 \%$ in total, depending slightly on the choice of $b_{\min }$. After transforming the impact parameter into a laboratory scattering angle according to Eq. 1 and summing up the various contributions, the differential cross section $d \sigma / d \theta$ is obtained, which is then convoluted with the angular resolution given above. The resulting angular distribution is compared with the data and the best value of $b_{\min }$ is determined by a $\chi^{2}$ minimization procedure. Within this analysis, only the overall normalization is adjusted, but is found to deviate from unity only within the systematic error. The analysis is restricted to scattering angles above $2 \mathrm{mrad}$ in order to minimize effects due to nuclear contributions (see above). An enhancement of the DGDR cross section for ${ }^{136} \mathrm{Xe}$ in comparison to the harmonic limit was observed in [2]. We repeated, therefore, the analysis increasing the DGDR cross section as calculated above by a factor 2 . 'The results given below do not change significantly because of the small fraction contributed by the DGDR to the total angular distribution.

The result from the $\chi^{2}$ analysis is given in Tab. I. The best values for $b_{\min }$ amount to $14.5 \pm 0.4 \mathrm{fm}$ and 13.6 $\pm 0.6 \mathrm{fm}$ for the $\mathrm{Pb}$ and $\mathrm{Sn}$ target, respectively. The corresponding calculated angular distributions are shown in Fig. 1. These sharp-cutoff $b_{\min }$ values derived from the experimental data should be compared with the $b_{\min }$ parametrizations mentioned in the introduction. A comparison with both the $\mathrm{BCV}$ parametrization [6] and that of Kox et al. [7] is provided in Tab. I. In case of the $\mathrm{Pb}$ target, the $b_{\min }$ value obtained with the $\mathrm{BCV}$ parametrization is in perfect agreement with the experimental result, the value obtained with the parametrization of 
TABLE I. Sharp-cutoff minimum impact parameters $b_{\min }$ as extracted from this experiment including statistical errors (first) and systematical errors (second). For comparison, the $b_{\min }$ values from the parametrizations suggested in [6] (BCV) and in [7] (Kox) are given. Values of $b_{\min }$ quoted for the soft-spheres model correspond to impact parameters for which the nuclear absorption amounts to 0.5 (see text).

\begin{tabular}{lll}
\hline \hline & Pb target & Sn target \\
\hline this experiment & $14.5 \pm 0.2 \pm 0.25$ & $13.6 \pm 0.3 \pm 0.35$ \\
BCV & 14.5 & 13.1 \\
Kox & 15.7 & 14.1 \\
soft spheres & 14.3 & 12.9 \\
\hline \hline
\end{tabular}

Kox et al. is too large. This finding is in accord to conclusions drawn in [5] from inclusive data for ${ }^{197} \mathrm{Au}$. In case of the $S n$ target, $b_{\min }$ values from both parametrizations are within the error limits of the experimental result. Statistical and systematical experimental errors are provided in the table, the latter ones are deduced from the absolute accuracy in scattering angle (see above).

As an alternative to a sharp cutoff $b_{\min }$ approximation, a 'soft-spheres' model was suggested and explored in [5]. Essentially, the sharp-cutoff is replaced by a smooth cutoff in impact parameter which is obtained by calculating the effect of nuclear absorption using an optical potential. The optical potential is constructed in eikonal approximation by folding the mass density distributions of target and projectile ('t $\rho \rho$ approximation', see [5]), from which finally the nuclear absorption probability is calculated. At each impact parameter, the cross section obtained from the semi-classical calculation is then multiplied by the probability that nuclear absorption does not occur. We use realistic density distributions described by modified Fermi functions which were adjusted to charge density distributions from elastic electron scattering; their parameters are given in the compilation by Barret and Jackson [15]. For the ${ }^{\text {nat }} \mathrm{Sn}$ targets, we use parameters averaged over the stable tin isotopes. Density distributions for ${ }^{136} \mathrm{Xe}$ are not available. Instead, we use that of the neighboring isotope ${ }^{138} \mathrm{Ba}$, scaling slightly the radius parameter according to an $A^{1 / 3}$ dependence. In any case, the radial shapes of neutron and proton distributions are assumed to be identical. For our scattering systems, the absorption probability computed with the soft-spheres model drops from a value of 0.9 to a value of 0.1 over a range of $2.1 \mathrm{fm}$, both for the $\mathrm{Pb}$ and $\mathrm{Sn}$ target. A value of 0.5 is reached at $b=14.3(12.9) \mathrm{fm}$ with the $\mathrm{Pb}(\mathrm{Sn})$ target (see also Tab.I), rather close to the $b_{\min }$ values of the $\mathrm{BCV}$ parametrization. It should be noticed that the BCV sharp-cutoff parametrization has been derived based on assumptions similar than those underlying the soft-spheres model.

The angular distributions obtained with the softspheres model arc shown in Fig. I. In case of the $\mathrm{Pb}$ tar- get, the description of the experimental data at largest angles is slightly improved compared to that obtained within the sharp-cutoff approximation. In case of the Sn target, the description is slightly worse because the equivalent $b_{\min }$ value of the soft-spheres model (see Tab. I) is lower than the extracted best value in sharp cutoff approximation. The main difference between the two models, however, i.e. a smooth or a sharp drop in cross section around grazing incidence, respectively, is masked to a large extent by the finite angular resolution in our measurement, in particular in case of the $S_{n}$ target. Nevertheless, we may conclude that the process of nuclear absorption can be described fairly well within the softspheres model on the basis of realistic mass density distributions.

In summary, we have measured the fragment angular distribution for the process of electromagnetic giant resonance excitations in heavy-ion collisions at high bombarding energy. The distributions can be well reproduced within the framework of the semi-classical approach. Minimum impact parameters were determined, which can be understood quantitatively describing nuclear absorption on the basis of realistic target and projectile mass density distributions.

This work was supported by the German Federal Minister for Education and Research (BMBF) under Contracts 06 OF 474 and $06 \mathrm{MZ} 864$ and by GSI via Hochschulzusammenarbeitsvereinbarungen under Contracts OF ELK, MZ KRK, and by the Polish Committee of Scientific Research under Contract PB2/P03B/113/09.

[1] T. Aumann, P. F. Bortignon, and H. Emling, Annu. Rev. Nucl. Part. Sci. 48, 351 (1998).

[2] R. Schmidt et al., Phys. Rev. Lett. 70, 1767 (1993).

[3] A. Winther and K. Alder, Nucl. Phys. A319, 518 (1979).

[4] C. A. Bertulani and G. Baur, Phys. Rep. 163, 299 (1988).

[5] T. Aumann, C. A. Bertulani, and K. Sümmerer, Phys. Rev. C51, 416 (1995).

[6] C. J. Benesh, B. C. Cook, and J. P. Vary, Phys. Rev. C40, 1198 (1989).

[7] S. Kox et al., Phys. Rev. C35, 1678 (1987).

[8] J. Cub et al., Nucl. Instr. and Meth. A402, 67 (1998).

[9] T. Blaich et al., Nucl. Instr. and Meth. A314, 136 (1992).

[10] V. Metag et al., Comments on Nucl. Part. Phys. 16, 213 (1986)

[11] K. Boretzky et al., Phys. Lett. B384, 30 (1996).

[12] W. J. Llope and P. Braun-Munzinger, Phys. Rev. C41, 2644 (1990).

[13] S.S. Dietrich and B.L. Berman, At. Data Nucl. Data Tables 38, 199 (1988).

[14] F. E. Bertrand, Ann. Rev. Nucl. Part. Sci. 26, 457 (1976).

[15] P. C. Barret and D. F. Jackson, "Nuclear Size and Structure", Oxford University Press, NY, 1977 